\title{
Toward a taxonomy of attention shifting: Individual differences in fMRI during multiple shift types
}

\author{
TOR D. WAGER \\ Columbia University, New York, New York \\ JOHN JONIDES \\ University of Michigan, Ann Arbor, Michigan \\ EDWARD E. SMITH \\ Columbia University, New York, New York \\ and \\ THOMAS E. NICHOLS \\ University of Michigan, Ann Arbor, Michigan
}

\begin{abstract}
Although task switching is often considered one of the fundamental abilities underlying executive functioning and general intelligence, there is little evidence that switching is a unitary construct and little evidence regarding the relationship between brain activity and switching performance. We examined individual differences in multiple types of attention shifting in order to determine whether behavioral performance and fMRI activity are correlated across different types of shifting. The participants $(n=39)$ switched between objects and attributes both when stimuli were perceptually available (external) and when stimuli were stored in memory (internal). We found that there were more switchrelated activations in many regions associated with executive control-including the dorsolateral and medial prefrontal and parietal cortices-when behavioral switch costs were higher (poor performance). Conversely, activation in the ventromedial prefrontal cortex (VMPFC) and the rostral anterior cingulate was consistently correlated with good performance, suggesting a general role for these areas in efficient attention shifting. We discuss these findings in terms of a model of cognitive-emotional interaction in attention shifting, in which reward-related signals in the VMPFC guide efficient selection of tasks in the lateral prefrontal and parietal cortices.
\end{abstract}

Over the last 15 years, neuroimaging studies have consistently identified a set of structures associated with the maintenance and manipulation of information and the control of attention. The function of this set of brain regions has been associated with a set of psychological processes described collectively as executive control operations, due to their central role in coordinating perceptual and motor processes in the service of internal goals. These mechanisms, broadly associated in traditional neuropsychology with the frontal lobes, allow us to focus on learning a novel task, listen to a friend when we have something else on our minds, notice and correct inappropriate behavior, and, possibly, even regulate our emotional responses (Posner \& Rothbart, 1998).

All major simulated cognitive architectures of which we are aware include an executive system or set of systems, although much work in the field has been directed toward

Correspondence concerning this article should be addressed to T. D. Wager, Department of Psychology, Columbia University, 1190 Amsterdam Ave., New York, NY 10027 (e-mail: tor@paradox.psych. columbia.edu). understanding whether there is a single executive system underlying performance on various tasks or whether separate domain-specific systems govern cognitive regulation (Anderson \& Lebiere, 1998; Baddeley, 1992; Meyer \& Kieras, 1997; Newell, 1992; Shallice \& Burgess, 1996). These architectures, most of them explicit computational simulations capable of modeling human performance, provide ideas about a number of candidate executive operations. These include the shifting of attention, the active maintenance of goals and information in working memory (WM), task scheduling and prioritization, and the mental manipulation of information in WM (e.g., rotating a mental image or updating and releasing stored items).

Although the importance of such control operations is virtually unquestioned, we are at the beginning stages of understanding the cognitive and brain mechanisms that underlie them. How many executive control systems are there, and what distinctions among control operations are the most meaningful? Are the candidate operations we have listed unitary, or can they be fractionated? In this study, we focus on attention shifting as a candidate control operation and ask whether performance on shifting 
tasks can be dissociated on the basis of three factors: (1) Among what representations is attention shifted? (2) In what locus are the items between which attention is shifted stored-in WM or in perceptual buffers? (3) And finally, when does the shift occur-when an actual stimulus is presented and a behavioral response is required, or in advance of stimulus presentation and response selection?

\section{What Representations and What Locus of Storage?}

Theories of switching have combined evidence on switch costs from studies varying the cue type (Coull, Frith, Buchel, \& Nobre, 2000), the target tasks (Allport, Styles, \& Hsieh, 1994; Rubinstein, Meyer, \& Evans, 2001), and whether switching occurs among the tasks themselves (i.e., both stimulus and response sets), operations (Spector \& Biederman, 1976), stimulus-response mapping rules (Rubinstein etal., 2001), objects stored in WM (Garavan, 1998), object attributes (Owen, Roberts, Polkey, Sahakian, \& Robbins, 1991; Rogers \& Monsell, 1995), or combinations of these. The question of consistency across types is critical if particular shifting tasks are used as measures of an executive ability.

In this study, we combined two types of evidence that have been considered for understanding the relationships between types of shifting: individual differences in performance and brain-imaging activation. In individualdifferences studies, the basic measures of performance are the reaction time (RT) and error rate costs for trials in which a shift of attention is required, as compared with trials with no shift (i.e., shift-no shift; see, e.g., Garavan, 1998; Gopher, Armony, \& Greenshpan, 2000; Hsieh \& Allport, 1994; Meiran, Chorev, \& Sapir, 2000; Monsell, Yeung, \& Azuma, 2000; Rogers \& Monsell, 1995; Rubinstein etal., 2001; Shafiullah \& Monsell, 1999; Yeung \& Monsell, 2003).

Individual-differences studies of performance test whether individuals who are relatively good (low shift cost) at one task are also good at another task, as measured by correlations in performance scores across participants. Such studies have demonstrated that attention shifting is moderately related to more complex tests of executive WM, which in turn are moderately predictive of yet more complex intelligence tests (Bleckley, Durso, Crutchfield, Engle, \& Khanna, 2003; Conway, Kane, \& Engle, 2003; Kane et al., 2004; Miyake etal., 2000; Salthouse, 1996; Salthouse, Fristoe, McGuthry, \& Hambrick, 1998). Some evidence indicates that basic processing speed is a contributor to both effective WM and attention shifting and that shifting tasks can be relatively highly intercorrelated when the same materials are used (Salthouse et al., 1998). However, previous studies have not, to our knowledge, systematically looked for subtypes of attention shifting. Although some of the variance in switching performance may be attributed to common factors that presumably reflect a more general executive system, at least as much of the variance typically remains unexplained. A difficult issue is that shift costs in performance reflect the final output of multiple mechanisms and processes, and it is easy for commonalities to be swamped by task-specific processes. It could be that common mechanisms would be readily apparent if we could peer directly into the unmeasurable intermediate stages of processing.

Neuroimaging affords such an opportunity to directly measure intermediate processing stages. Here, we use functional magnetic resonance imaging (fMRI) to characterize task switching in terms of the brain regions that become active during shifts of attention, as compared with matched nonshift trials. To our knowledge, over 30 studies of attention shifting have been published to date, although few studies have been performed to investigate different types of shifting in the same participants. These studies have collectively provided new insights into the question of the relationships among executive functions. Different studies have reported somewhat different areas of activation, depending on the particulars of the task, analysis, and group of participants; however, meta-analysis has revealed that a core set of frontal, parietal, and temporal cortical regions is consistently activated by shifting tasks (Wager, Reading, \& Jonides, 2004). These regions are a subset of those engaged by executive WM tasks, supporting the view that executive WM involves a heterogeneous collection of cognitive operations that includes attention shifting. Although these regions are reliably activated across different kinds of shifting tasks, little is known about which of these regions contribute to good behavioral performance (i.e., low switch costs) and whether different regions are related to performance in systematic ways in different shifting tasks. These two questions motivated the present study.

In the present experiment, we contrasted four types of shifts of attention that varied on two factors. The first factor was the type of representation switched (type-ofrepresentation): Participants switched between whole objects on some trials and between attributes (i.e., dimensions) of objects on other trials. The second factor, locusof-representation, was the perceptual availability of stimuli. On some trials, participants switched between visible stimuli (the external condition), and on other trials, participants switched between stimuli stored in working memory (the internal condition). Both types of switching have been tested behaviorally and with neuroimaging (Garavan, 1998; Sylvester et al., 2003), but differences in shifting costs depending on the locus of representation have not been systematically tested.

In addition, we identified participants who showed high or low switch costs overall (good or poor switchers) and asked which brain switch costs were correlated with behavioral performance. Thus, performance could be correlated with switching activations for attribute or object switching with internal or external stimuli or with some combination of these.

\section{When Does the Shift Occur?}

A third factor concerns when switching processes are performed or, more specifically, whether switching pro- 
cesses are performed in advance of seeing a target and engaging specific response selection mechanisms. We investigated this issue on a subset of trials (object-switching trials) in our task (see Figure 1). The task is described in detail in the Method section, but a brief explanation here will illustrate the point. Participants are first given a cue that tells them which object to focus on. That information is not sufficient to make a response, however, since the object has two attributes and the participants do not yet know which will guide their response. Following a cue-target interval (CTI), the remaining information about the attribute is presented, and the participants press the appropriate response button. A number of behavioral studies have documented that some, but not all, of the switch cost can be eliminated by a long (e.g., 1,200-msec) CTI (Mayr, 2003; Mayr \& Kliegl, 2000; Meiran, 1996; Rogers \& Monsell, 1995; Rubinstein et al., 2001). A residual switch cost is still apparent after prolonged intervals and does not diminish further with time, suggesting that some (but not all) task-switching processes can be configured in advance of the stimulus. These advance processes may be related to selecting the appropriate mental set or task goal (e.g., respond to the red object), and they have been called endogenous (Mayr, 2003; Mayr \& Kliegl, 2000; Meiran, 1996; Rogers \& Monsell, 1995). Here, we refer to the time used in advance configuration as task set engagement time, and to the associated shift costs as set shift costs. The processes of selecting a specific rule and motor response (e.g., rectangle $\rightarrow$ right button) have been referred to as rule activation, and the associated residual switch costs as exogenous switch costs, because they reflect processes that must be cued by an imperative stimulus. We refer to this time interval as rule engagement time and the associated costs as residual shift costs. Thus, surprisingly, completely shifting attention to a particular object associated with several rules requires actually selecting and executing a specific rule. Task set engagement can allow for partial selection of the object, but even given ample time to select the object, the selection process requires an additional rule engagement period to complete.

In our study, the CTI was self-paced. The participants were instructed to respond as quickly as they could to the cue by shifting their attention appropriately and pressing a button when ready to continue, similar to the readiness response time measured by Meiran and colleagues (Meiran, Hommel, Bibi, \& Lev, 2002). ${ }^{1}$ When they pressed the button, the target stimulus appeared immediately. Thus, for object switches, we can obtain two performance measures: first, the set shift costs in the time taken to respond to the cue and, second, residual object switch costs. We can then ask whether object-switching-related brain activations are correlated with task set engagement time, rule engagement time, or both. Brain areas that show each corre-

A

Attribute switch (if different)

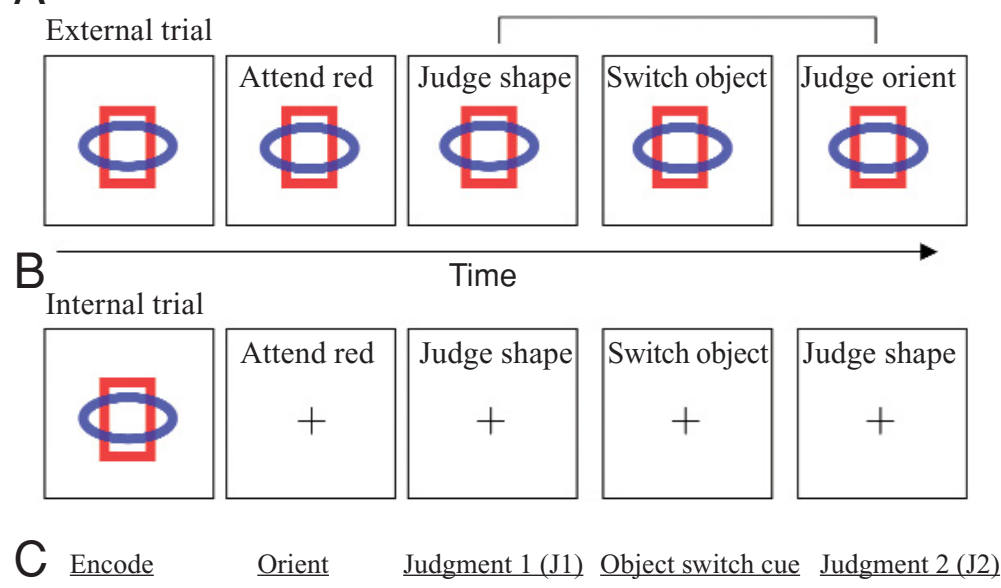

Figure 1. Example trials from the task design (not drawn to scale). The panels illustrate successive events within the trial. The objects were drawn in blue and red. The eight different configurations of red and blue ellipses and rectangles in vertical and horizontal orientations were used in equal numbers, with a new stimulus configuration appearing on each trial. (A)On external trials, the stimulus was visible continuously, as is depicted here. (B) On internal trials, the stimulus was visible only in the first panel, and language cues appeared in the center of the screen. (C) The names of each phase of the trial are printed below the panels. $\mathrm{Ob}$ ject-switching reaction time (RT) costs in the object switch cue phase were calculated as RT for switch-stay trials. Trials were attribute switches if the attribute for the second judgment (J2) did not match that for the first judgment (J1). For J2, RT measures were collected for each trial type (switch attribute and object, switch object only, switch attribute only, or no switch). 
lation are likely to be related to both goal selection and response selection processes, respectively. Attributeswitching performance costs reflect both processes, so we expect them to correlate with brain regions that perform both set-shifting and rule engagement processes.

To the degree that switching is a unitary construct or ability, we would expect to find positive correlations between brain and behavioral switch costs across different types of switching and in the same brain regions. Alternatively, some subtypes of switching could emerge as distinct but coherent constructs; for example, we might expect behavioral performance to predict lateral prefrontal activation only in conditions that involve task set engagement (i.e., cue switch costs for objects and switch costs for attributes, across external and internal conditions). Finally, all four types of switching could share no underlying commonalities in performance or brain activation, suggesting that there are many separate types of attention shifting.

The results we present here suggest that there is a significant commonality among all the types of shifting studied, implying that there is a general ability underlying them, although some regions seemed to correlate with only a subset of switch types and to be activated preferentially by some switch types. That is, different types of switching involve some, but not all, of the same component processes.

\section{METHOD}

\section{Participants}

The participants were 43 right-handed adults, $18-40$ years of age, recruited from the Ann Arbor area. All the participants voluntarily signed informed consent documents, and the study was approved by the University of Michigan Institutional Review Board. The participants were selected from the extreme ends (top and bottom $25 \%)$ of a larger sample $(N=268)$ on the basis of overall switch costs across conditions (Wager \& Smith, 2005). Selection was performed as behavioral data were collected on a rolling basis. In this way, we recruited 22 individuals with particularly high switch costs and 21 with particularly low switch costs (all fMRI analyses were performed using actual measured switch costs, rather than high-low group assignment). All the participants reported no history of psychiatric illness or major depression and were asked to refrain from alcohol use for $48 \mathrm{~h}$ prior to fMRI scanning. Two participants were excluded due to excessive head motion ( $>6 \mathrm{~mm}$ total range across the experiment or $>4 \mathrm{~mm}$ range within a scanning run), one due to poor spatial normalization to the anatomical Montreal Neurological Institute (MNI) T1 template, and one as a multivariate outlier in RT performance across behavioral and fMRI sessions. This left a final sample of $n=39$ ( $n=19$ low and $n=20$ high).

The participants were paid $\$ 50$ for participation, in addition to a performance bonus calculated on the basis of both speed and accuracy. Bonus feedback was given after blocks of 48 trials, with 5 cents awarded per correct trial only if the participants' mean RT was shorter than that on the previous block. To receive the bonus on the first block, the participants had to be faster than $1,000 \mathrm{msec}$, on average. Cumulative bonuses ranged from approximately $\$ 6$ to $\$ 12$.

\section{Task Design}

The task consisted of multipart trials, each requiring two judgments about the same stimulus. The stimuli were images of two overlapping objects, one red and one blue, shown in the first panel of Figure 1A. The color served as a cue for which object to attend.
Each object was either an ellipse or a rectangle (two rectangles or two ellipses were allowed). One object was always oriented vertically, and the other horizontally.

On each self-paced trial, the objects appeared during an initial encoding phase, during which the participant was instructed to focus on the stimulus and prepare for the trial. When the participant pressed the space bar, a cue appeared above the objects with the words "attend red" or "attend blue," which signaled to the participant which object should be attended (orient phase). Upon another space bar press, a judgment was required (J1 phase): either the words "judge shape" or the words "judge orientation" appeared. If shape judgment was indicated, the participant pressed with the right index finger if the attended shape was an ellipse or with the right middle finger if it was a rectangle. If an orientation judgment was indicated, the participant pressed with the right index finger if the attended shape was vertical or with the right middle finger if it was horizontal.

Following the buttonpress and an additional 200-msec delay, the words "switch object" or "stay object" appeared on the screen, indicating whether the participant was to shift attention to the unattended object or continue attending the same object (object switch cue phase). The participant was instructed to press the space bar when he or she completed the shift of attention. After the response and the 200-msec delay, the participant was asked to either "judge shape" or "judge orientation" again (J2 phase).

The switch variable. Attribute switch trials were ones in which the relevant attribute (shape or orientation) for the second judgment differed from that for the first judgment; the critical RT measure for this was during the second judgment period for switch and nonswitch trials. Object switch trials were ones on which the participant was asked to shift between objects, and two RT measures for this shift were collected: time to respond to the switch cue "stay" versus "switch" and time to respond during the second judgment. Previous research has shown that even given quite a long time to prepare for an upcoming switch trial, responses to targets are still slower on switch than on no-switch trials (e.g., Meiran etal., 2000; Monsell et al., 2000; Rogers \& Monsell, 1995).

The locus of representation (internal/external) variable. On external trials (Figure 1A), the stimulus remained on the screen throughout the trial, and cues appeared just above the stimulus. On internal trials (Figure 1B), the stimulus was present only for the initial viewing period, so that it was necessary to refer to a representation of the object in WM to complete the trial. When the words "attend red" (or blue) appeared, the shape disappeared for the rest of the trial. Blocks of 48 external (E) and internal (I) trials were alternately performed (E I E I E I), with two blocks of practice preceding the test blocks.

The trials were arranged in a fixed prerandomized order to minimize order effects on individual differences (e.g., Miyake et al., 2000). In the fMRI session, trial ordering was optimized using a genetic algorithm (Wager \& Nichols, 2003). Since there is a tradeoff between the efficiency of detecting main effects of object and attribute switching and estimating the shape of the hemodynamic response evoked by switching, we optimized the design for an equally weighted combination of the two. This choice ensured that we would have relatively high power to detect main effects and link them with some specificity to the switch events.

\section{Behavioral Data Analysis}

Mean RTs (in milliseconds) for correct trials in each condition were calculated for each participant after removing outliers greater than three standard deviations from the mean of the condition within participants (Kane \& Engle, 2003). The critical comparisons were (1) comparing switch trials with nonswitch trials during J2 for each of the four switch types (internal/external $\times$ attribute/object), and (2) comparing object switch with nonswitch RTs in the object switch cue period. Mean RTs in J2 were analyzed for main effects of object switching, attribute switching, internal versus 
external task, and all interactions in a $2 \times 2 \times 2$ factorial repeated measures analysis of variance (ANOVA) design. RTs in the object cue period were analyzed in a 2 (object switch vs. nonswitch) $\times 2$ (internal vs. external) repeated measures ANOVA. Analyses on log RTs produced qualitatively identical results.

Because we were interested in identifying brain differences that distinguish good and poor switchers overall, we selected participants on the basis of their overall switch times across conditions For purposes of correlating performance with fMRI activity, we averaged switch costs across the four switch types in $\mathrm{J} 2$ to obtain an overall measure of performance costs ( $\mathrm{J} 2$ switch costs). We averaged the object-switching costs during the cue interval (cue switch costs) to obtain a behavioral measure related to task set preparation in the object conditions. Average switch costs showed a higher overall test-retest reliability than any single switch cost did. Reliability was calculated across an interval of 1 week to several months between preliminary and fMRI testing; reliability values are reported in the Results section.

\section{fMRI Acquisition and Analysis}

Acquisition and preprocessing. Spiral GRE functional images were acquired on a GE Signa $3 \mathrm{~T}$ scanner at $\mathrm{TR}=1.5, \mathrm{TE}=20$, Flip $=90,64 \times 64$ matrix, $3.75 \times 3.75 \times 5 \mathrm{~mm}$ voxels, skip 0 . Twenty-six slices provided whole-brain coverage. Prior to analysis, images were corrected for differences in slice acquisition time, using 4-point sinc interpolation (Oppenheim, Schafer, \& Buck, 1999), and head movement, using MCFLIRT (Jenkinson, Bannister, Brady, \& Smith, 2002). A high-resolution spoiled GRASS image was collected and spatially normalized to the MNI template (avg152t1.img), using SPM99 (Ashburner \& Friston, 1997). Spatial normalization transformations and 9-mm FWHM isotropic Gaussian smoothing were applied to all functional images prior to analysis.

Individual participant models. Analysis for each participant was done using the general linear modeling (GLM) framework implemented in SPM2, with a canonical hemodynamic response function convolved with onset indicator vectors for each switch type (no switch, attribute switch only, object switch only, or double switch, crossed with internal and external conditions). Object switches were performed in two stages: in response to the object switch cue (advance preparation component) and when the second judgment was made during the trial (residual switch component). These two phases always occurred proximally in time, so predicted object-switching BOLD activity for the two phases was highly correlated $(r=.95)$. Thus, one regressor was used to capture activity in both periods. The onset of the second judgment period (the instruction to "judge shape" or "judge orientation") was used as the onset of the predicted hemodynamic response for switch and nonswitch predictors.

A high-pass filter cutoff of $1 / 180 \mathrm{~Hz}$ for all sessions and all participants was chosen on the basis of cumulative spectral power $\times$ frequency plots; less than $5 \%$ of the power in all contrasts of interest for all the participants was removed by the filter. No global scaling (Della-Maggiore, Chau, Peres-Neto, \& McIntosh, 2002; Gavrilescu etal., 2002) and no low-pass filtering were used.

Contrasts of main effects for switch versus nonswitch trials for each switch type (fMRI switch cost) were calculated for internal and external blocks. The contrasts were internal object (IO) switch, internal attribute (IA) switch, external object (EO) switch, and external attribute (EA) switch. For each contrast, event-related activity in switch trials was compared with that in nonswitch trials.

Group analysis: Brain-behavior correlations. Following estimation of fMRI switch costs for each participant, group analyses were performed on contrast values in gray matter voxels. We conducted a second-level mixed GLM analysis to determine whether switch costs in each brain voxel were significantly predicted by behavioral performance measures. The analysis was a 2 (between) $\times 4$ (within) mixed effects design. The two between-subjects factors were mean-centered behavioral costs: overall switching RT cost in J2 and object-switching cost in the cue period. Repeated measures were the four within-subjects factors of brain switch costs (IO, IA, EO, and EA). Using this model, we were able to estimate six brain-behavior correlations of interest: J2 cost with an IO, IA, EO, and IA and cue switch costs with an IO and EO. These are listed in the first column of Table 1. Dependencies between switch costs were estimated using SPM2, as appropriate for a repeated measures analysis.

We first performed an omnibus $F$ test to locate voxels with significant switch costs overall ( $p<.05$, FDR corrected; Genovese, Lazar, \& Nichols, 2002). Where the omnibus test was significant, we proceeded to examine the pattern of correlations and interpret the results.

How might we interpret brain-behavior correlations that are significant for some switch costs, but not for others? The correlations we might expect to be significant for brain shift costs related to type of representation, locus of representation, and set versus rule engagement are shown in Table 1. Set-shifting regions should show positive correlations between object-switching costs in the cue period, a behavioral measure of task set engagement time, and both IO and EO brain measures. These regions should also show positive correlations between J2 switch costs (behavior) and attribute-shifting costs (brain), since both measures contain a task set engagement component. Rule engagement regions should show a different pat-

Table 1

Switch-Related Contrasts of Interest and the Switch-Related Processes They Are Likely to Reflect Switch-Related Contrasts of Interest and the Switch-Related Processes They Are

\begin{tabular}{|c|c|c|c|c|c|c|}
\hline \multirow[b]{4}{*}{ Contrast } & \multirow{3}{*}{\multicolumn{2}{|c|}{ Process (When) }} & \\
\hline & & & \multirow{3}{*}{\multicolumn{2}{|c|}{$\begin{array}{c}\text { Type of } \\
\text { Representation } \\
\end{array}$}} & \multicolumn{2}{|c|}{$\begin{array}{c}\text { Locus of } \\
\text { Representation }\end{array}$} \\
\hline & & & & & \multirow[b]{2}{*}{$\begin{array}{r}\text { Perception } \\
\text { (External) }\end{array}$} & \multirow{2}{*}{$\begin{array}{l}\text { Working } \\
\text { Memory } \\
\text { (Internal) }\end{array}$} \\
\hline & $\begin{array}{c}\text { Task Set } \\
\text { Shift }\end{array}$ & Rule Shift & & & & \\
\hline Object switch external in cue period & $\checkmark$ & & $\checkmark$ & & $\checkmark$ & \\
\hline Object switch internal in cue period & $\checkmark$ & & $s$ & & & $\checkmark$ \\
\hline Object switch external in J2 & & $d$ & 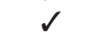 & & $\checkmark$ & \\
\hline Object switch internal in J2 & & $\checkmark$ & $\checkmark$ & & & $\checkmark$ \\
\hline Attribute switch external in $\mathrm{J} 2$ & $\checkmark$ & 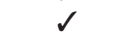 & & $\checkmark$ & $\checkmark$ & \\
\hline Attribute switch internal in J2 & $\checkmark$ & $\checkmark$ & & $\checkmark$ & & $\checkmark$ \\
\hline
\end{tabular}

Note-The first column describes the contrast. The second and third columns indicate whether the contrast is likely to reflect task set shifting (i.e., part or all of the switch cost for this contrast involves preparation of a new task set) and/or rule shifting (i.e., part of the switch cost measured involves residual shift costs after the task set has been selected). $\mathrm{J} 2$ is the second judgment period in the trial. The subsequent columns describe the type of representation associated with each shifting contrast (object or attribute) and the locus of representation (perception or working memory-i.e., external or internal) 
Table 2

Regions Showing Significant Brain-Behavior Correlations $(p<.05$ Corrected) in the Omnibus Analysis

\begin{tabular}{|c|c|c|c|c|c|}
\hline \multirow[b]{2}{*}{ Region } & \multicolumn{3}{|c|}{ Coordinates } & \multirow[b]{2}{*}{$z$ Score } & \multirow[b]{2}{*}{ Voxels* } \\
\hline & $x$ & $y$ & $z$ & & \\
\hline Right superior cerebellar hemisphere & 30 & -68 & -30 & 4.01 & 62 \\
\hline Left anterior superior cerebellum & -22 & -38 & -35 & 3.89 & \\
\hline Left inferior temporal gyrus & -41 & -19 & -35 & 3.77 & 26 \\
\hline Left inferior temporal gyrus & -49 & -26 & -25 & 5.72 & 97 \\
\hline Ventromedial prefrontal cortex & 0 & 22 & -15 & 7.64 & 2,083 \\
\hline Pregenual anterior cingulate & 4 & 45 & -5 & 4.58 & \\
\hline Right ventral anterior insula & 26 & 22 & -20 & 6.56 & \\
\hline Right inferior temporal gyrus & 56 & -34 & -25 & 5.03 & 141 \\
\hline Left fusiform & -45 & -56 & -15 & 4.73 & 114 \\
\hline Left extrastriate cortex & -38 & -90 & -10 & 4.85 & 167 \\
\hline Left visual cortex & -4 & -86 & -10 & 4.52 & 308 \\
\hline Right visual cortex & 11 & -82 & -10 & 4.85 & \\
\hline Hypothalamus & 4 & -4 & -10 & 4.28 & 62 \\
\hline Left supramarginal gyrus & -64 & -45 & 15 & 4.55 & 246 \\
\hline Left extrastriate cortex & -49 & -79 & 15 & 3.68 & 26 \\
\hline Left intraparietal sulcus & -45 & -56 & 50 & 7.46 & 3,507 \\
\hline Left anterior intraparietal sulcus & -45 & -41 & 55 & 5.81 & \\
\hline Precuneus & 4 & -90 & 30 & 6.02 & \\
\hline Left precuneus/intraparietal sulcus & -8 & -79 & 45 & 7.04 & \\
\hline Left parietal cortex & -34 & -75 & 40 & 7.64 & \\
\hline Left dorsolateral prefrontal cortex & -45 & 30 & 35 & 7.04 & 2,857 \\
\hline Left posterior middle frontal gyrus & -45 & 8 & 50 & 7.58 & \\
\hline Left superior frontal sulcus & -22 & 38 & 50 & 7.40 & \\
\hline Left superior frontal gyrus & -19 & -8 & 55 & 4.73 & \\
\hline Right medial frontal gyrus & 11 & 15 & 60 & 5.51 & 1,705 \\
\hline Right medial prefrontal cortex & 8 & 41 & 35 & 5.78 & \\
\hline Right intraparietal sulcus & 34 & -75 & 45 & 5.63 & 290 \\
\hline Right parietal cortex & 15 & -41 & 55 & 3.86 & 44 \\
\hline Left sensorimotor cortex & -30 & -30 & 70 & 3.86 & 26 \\
\hline
\end{tabular}

Note-Regions with empty voxel counts are contiguous with the regions listed above them. *Standardized $2 \times 2 \times 2$ voxel equivalent. Note that the threshold is not extent based and that regions consisting of 1 or more voxels meet the corrected threshold.

tern, shown in the third column of Table 1 . These regions should show relationships between behavioral and brain measures that have a rule engagement component. Regions related to efficient rule engagement should show negative correlations between $\mathrm{J} 2$ performance costs and IO, IA, EO, and EA brain switch activations. Conversely, regions consistently related to inefficient rule engagement should show positive correlations.

We were particularly interested in whether we could find some brain areas that showed significant brain-behavior correlations for all switch types, which would suggest sites for a common mechanism underlying general switching, and brain areas that showed significant correlations with a coherent set of brain switch costs (e.g., all correlations that contain a task set preparation component). Because correlations are highly susceptible to the influence of outliers, we conducted all analyses, after the omnibus test, with iteratively reweighted least squares (IRLS), a multiple regression technique relatively insensitive to the effects of outliers (Wager, Keller, Lacey, \& Jonides, 2005).

We used conjunction analyses to locate individual voxels that showed evidence for significant correlations in multiple shift types. This analysis tests whether a voxel is active in all shifting contrasts within a set (e.g., all those with a rule engagement component), and it was performed by thresholding individual robust correlation maps at $p<.05$ and finding the intersection of all thresholded maps.

Regions-of-interest analyses. We identified regions of interest that were contiguous "blobs" of significant voxels in the omnibus test. Because some contiguous significant regions appeared to be separate activation foci joined by the blurring effects of BOLD contrast and smoothing, we used hierarchical clustering on the coordinate locations to separate contiguous clusters into distinct subsets, where appropriate.

Within each region, we computed robust brain-behavior correlations for each switch type and examined the pattern of significant effects (Table 3). For example, a region might show significant brain-behavior correlations only for attribute switching or only for internal object switching. Examining these patterns can help us understand the differences among different switch types that lead to relatively modest correlations in behavioral performance across various switching tasks.

To count a correlation as significant in a region, we required that at least 3 voxels in the region were significant at $p<.05$, uncorrected. In Table 3, we report the maximum robust correlation values for each switch type in each region. If there were both positively and negatively correlated voxels in a region, both are reported.

\section{RESULTS}

\section{Behavioral Results}

Figure 2 shows mean RTs for high switch cost (Figure 2A) and low switch cost (Figure 2B) participants for internal and external switching of objects and attributes during fMRI scanning $(n=42)$. Error bars represent $95 \%$ 
Table 3

Regions Significant in the Omnibus Analysis

\begin{tabular}{|c|c|c|c|c|c|c|}
\hline & & & & Rule (Resid & dual) Shift & \\
\hline & & Task Set $(\mathrm{G}$ & Goal) Shift & & & \\
\hline & Objec & t-Cue & Attr & ibute & Object-1 & Residual \\
\hline & External & Internal & External & Internal & External & Internal \\
\hline Consistent Negative Correlations With $\mathrm{I}$ & Rule Shiftir & & & & & \\
\hline Ventromedial prefrontal cortex & -.45 & -.46 & -.55 & -.38 & $.53 /-.36$ & -.43 \\
\hline Pregenual anterior cingulate & -.56 & -.39 & -.53 & -.44 & $.33 /-.50$ & -.46 \\
\hline Consistent Positive Correlations With Te & ask Set Shi & ifting & & & & \\
\hline Left anterior intraparietal sulcus & .44 & $.47 /-.32$ & .37 & .49 & & -.45 \\
\hline Positive Correlations With Internal/Task & k Set Objec & ct Shifting & & & & \\
\hline Left fusiform & .48 & .49 & & .40 & & -.43 \\
\hline Left visual cortex & .60 & .43 & & .33 & & \\
\hline Right visual cortex & .63 & .46 & & .38 & & \\
\hline Left intraparietal sulcus & .73 & .54 & & .53 & .32 & -.41 \\
\hline Precuneus & .43 & .54 & -.33 & .41 & .32 & -.49 \\
\hline Left precuneus/intraparietal sulcus & .55 & .45 & -.34 & .51 & & -.49 \\
\hline Left parietal cortex & .73 & .50 & -.54 & .46 & & -.61 \\
\hline Left dorsolateral prefrontal corte & .60 & .65 & $.36 /-.36$ & .58 & .39 & -.41 \\
\hline Left posterior middle frontal gyrus & .59 & .71 & & .56 & .38 & -.46 \\
\hline Left superior frontal gyrus & .51 & .48 & & .57 & & -.62 \\
\hline Right medial frontal gyrus & $.44 /-.34$ & .61 & -.58 & $.39 /-.34$ & & -.45 \\
\hline Right intraparietal sulcus & .57 & 40 & -.32 & .57 & & -.51 \\
\hline Right superior cerebellar hemisphere & & .34 & -.35 & .58 & & \\
\hline Left anterior superior cerebellum & & .49 & & .49 & .36 & -.40 \\
\hline Left superior frontal sulcus & & .74 & -.39 & .48 & & -.40 \\
\hline Right medial prefrontal cortex & -.44 & .51 & -.39 & .32 & & -.58 \\
\hline Additional Regions & & & & & & \\
\hline Left inferior temporal gyrus & & -.38 & & -.53 & .37 & -.44 \\
\hline Left extrastriate cortex & -.38 & .34 & -.52 & & & -.45 \\
\hline Right medial prefrontal cortex & -.44 & .51 & -.39 & .32 & & -.58 \\
\hline Left sensorimotor cortex & & -.33 & & & & -.46 \\
\hline Right ventral anterior insula & & .47 & -.49 & $.37 /-.39$ & .34 & -.58 \\
\hline Right inferior temporal gyrus & .37 & & & .49 & .34 & -.49 \\
\hline Hypothalamus & & & -.43 & & .45 & -.45 \\
\hline Left supramarginal gyrus & .42 & -.46 & -.43 & -.42 & .51 & -.45 \\
\hline Left extrastriate cortex & & & -.34 & & & -.41 \\
\hline Left inferior temporal gyrus & & & & & .33 & -.41 \\
\hline Right parietal cortex & -.37 & & & .58 & & -.49 \\
\hline
\end{tabular}

Note-Table entries list significant robust IRLS correlation coefficients between brain and behavioral switch costs. Nonsignificant correlations are indicated by empty spaces.

confidence intervals based on average within-subjects switch costs.

During the object switch cue period (left panel of Figures $2 \mathrm{~A}$ and $2 \mathrm{~B}$ ), the participants were slower to press the space bar on switch trials than on nonswitch trials for both external and internal switches [mean cost $=137$ and $130 \mathrm{msec}$, respectively; $F(1,41)=15.50$ and 14.31 , $p<.001$ for each]. Since high switch cost and low switch cost participants were chosen from the extreme ends of the distribution, analyses within group were less informative than the analyses for the whole group; however, notably, even those with the lowest switch costs were still slower or approximately as fast on switch trials in each condition.

During J2, both switching objects and switching attributes significantly increased RTs. Costs were 80/157 msec for external/internal object switching and 81/109 msec for external/internal attribute switching [for object switching, $F(1,41)=88.5, p<.001$; for attribute switch- ing, $F(1,41)=25.2, p<.001$; each cell also showed highly significant switch costs]. Object and attribute switching interacted, with dual-switch trials taking particularly long, only in the internal task [58-msec interaction; internal/external $\times$ object $\times$ attribute switching, $F(1,41)=12.7$, $p=.001]$. In a test of only external RTs, the interaction was not significant $(-13 \mathrm{msec}, F<1)$.

Analyses of error rates showed the same pattern of additive decrements in performance for both attribute and object switching and an interaction such that dual-switch costs were higher for internal switching. This pattern indicates a lack of overall speed-accuracy tradeoff, although these are not shown, for reasons of space. Accuracy ranged from $92 \%$ (on internal double-switch trials) to $98 \%$ (on external no-switch trials).

Because the participants were chosen on the basis of overall switch costs, which induces positive correlations among individual components, we will not focus on correlations among behavioral switch costs. Overall J2 
A High switch cost group
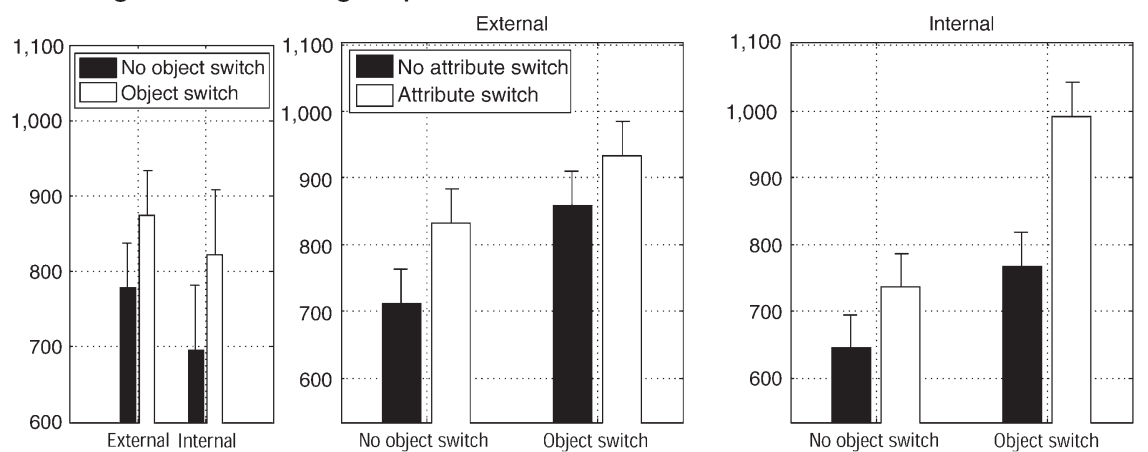

B Low switch cost group
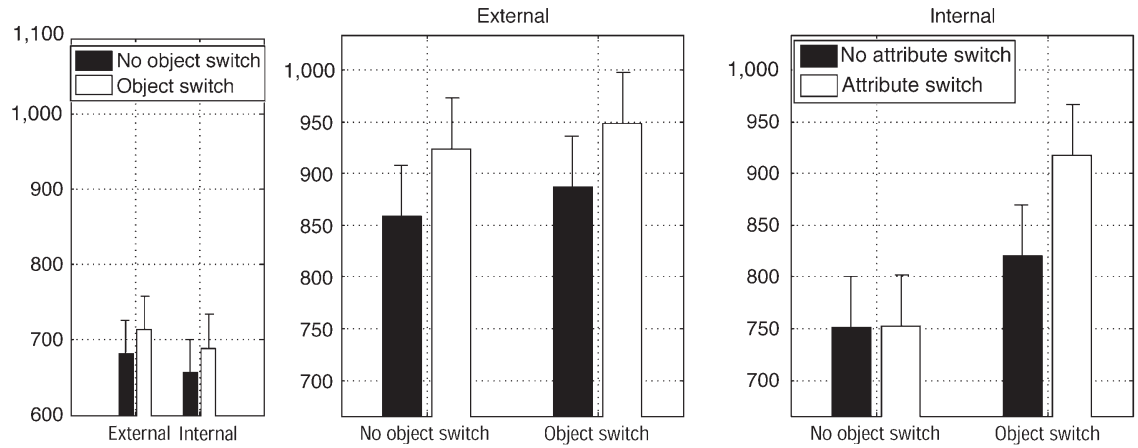

C Reliability of overall switch costs

\section{Cue vs. judgment switch costs}
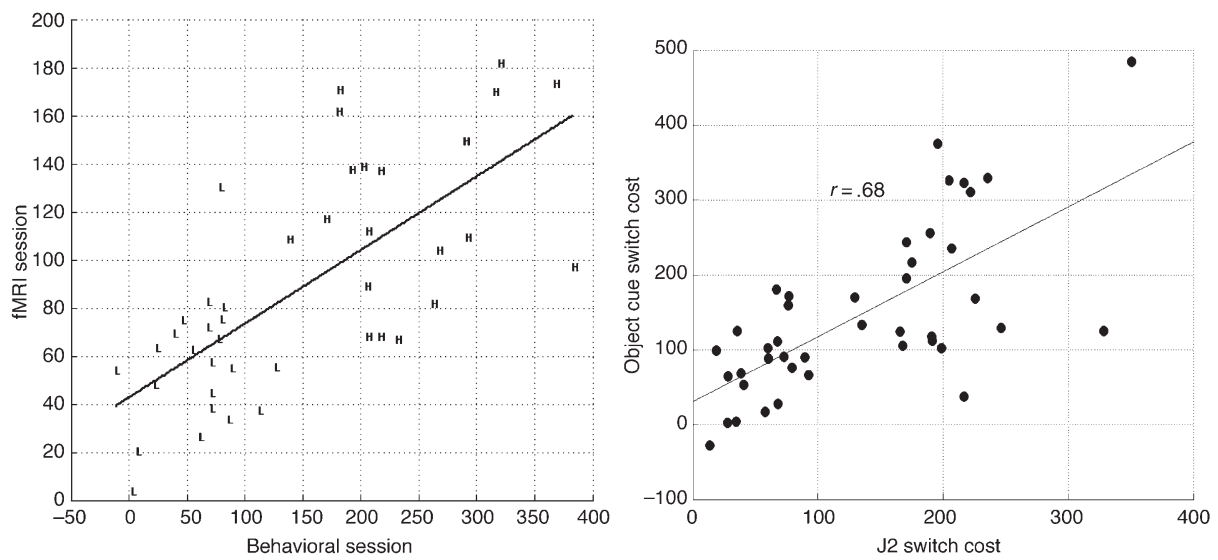

Figure 2. (A) Behavioral performance data for the high switch cost group. Left panel: Reaction times (RTs) by switching condition during the object switch cue period; object switches took longer during this period. Right panels: RTs during the second judgment period (when both attribute and object switches occurred) in the external and internal tasks, respectively; both attribute switching and residual object switching produced RT costs. (B) Behavioral performance data for the low switch cost group, as above. Switching was costly even for the best switchers. Accuracy data show costs of attribute and object switching for both groups (not shown for space reasons). Error bars show within-subjects $95 \%$ confidence intervals based on the average main effect of switching attribute and object (Loftus \& Masson, 1994). (C) Overall switching costs in the initial testing session ( $x$-axis) and fMRI session ( $y$-axis); high and low switch cost participants are labeled with $H$ and $L$, respectively. (D) Average switch costs in judgment ( $\mathrm{J} 2$ period, $x$-axis) plotted against object cue switch costs ( $y$-axis), averaging across behavioral and fMRI sessions. All fMRI analyses were conducted using the average switch cost across the two sessions, rather than high-low group labels, and all analyses were performed using multiple regression at the second level to find independent effects of cue switch and $\mathbf{J} 2$ switch costs. 
switch costs showed a high odd-even split half reliability $(r=.99)$ and a reasonable test-retest reliability across a period of weeks to several months $(r=.72$, Figure $2 \mathrm{C})$. Individual switch cost test-retest reliabilities were .63 and .69 for internal and external cue object switch costs, .45 and .44 for internal and external J2 object switch costs, and .53 and .53 for internal and external J2 attribute switch costs.

Overall J2 switch costs were positively correlated with switch cue costs $(r=.68, p<.05$; Figure $2 \mathrm{D})$, paralleling the findings in Meiran et al. (2002) that increased preparation time led to longer subsequent RTs but fewer errors. They also found that preparation produced a small decrease in switch costs. On the basis of Meiran et al.'s results and ours, it is likely that a participant may show high switch costs in preparation times because of multiple processes: (1) inefficient task preparation, which requires more time to complete to some subjective criterion level before proceeding, and (2) greater preparatory activity in advance of viewing the target, a strategic shift in the criterion for indicating readiness that is expected to decrease shift costs in the $\mathrm{J} 2$ period. The strong positive correlation between cue shift costs and $\mathrm{J} 2$ shift costs is evidence that the first effect predominates.

High-low group classifications for each participant are shown as "H" (high) and "L" (low) in Figure $2 \mathrm{C}$; however, we reasoned that the measured switch cost is a more accurate predictor of switching ability than high/low group membership, and thus, all fMRI analyses were performed using $\mathrm{J} 2$ switch costs and cue switch costs as simultaneous continuous predictors in multiple regression. In these multiple regressions, brain regions showing positive relationships with both cue and $\mathrm{J} 2$ switch costs are related to both preparatory and residual switch costs, each for the other. Thus, in such areas, a relatively high percentage of the variance in brain switch costs may be explained by a combination of performance switch costs in preparatory and posttarget switching.

\section{fMRI Results}

Event-related switching activations. This report is concerned mainly with individual differences in shifting attention. However, to address the issue of whether individual-differences effects occur in areas activated by switching attention, we first identified regions that were more active in shift versus nonshift trials in each of the four switch types (IO, EO, IA, and EA). To identify the most strongly activated regions, we identified voxels that were independently activated in at least two shift types at a threshold of $p<.05$ (corrected for gray matter volume, using SnPM with 10-mm variance smoothing). The results, shown in Figure 3 and in Table4, identified activation in all regions associated with task switching in our previous meta-analysis, including the bilateral intraparietal sulcus (IPS), the premotor/superior frontal sulcus (SFS), the anterior cingulate, the precuneus, and the left inferior temporal/occipital cortex. Our results also showed activations in the left dorsolateral prefrontal cortex (DLPFC), the bi- lateral cerebellum, and the striate and extrastriate cortices. All of these have been found in other individual studies of attention shifting but did not reach corrected significance in the meta-analysis. Parietal activations at this stringent threshold (Figure 3A) were predominantly left lateralized, but at slightly lower thresholds $(p<.001$ in two or more switch types; Figure 3B), activity was bilateral. Also activated at lower thresholds were the left anterior insula and the thalamus (confirming a prediction from the meta-analysis), the bilateral putamen, the hippocampus, and the bilateral extrastriate cortex.

Within voxels that responded to more than one switch type, we classified voxels as common (showing no differences among switch types) or as preferentially responsive to some switch types. To do this, we performed a mixed 2 (behavioral switch costs, between subjects) $\times$ 2 (object vs. attribute, within subjects) $\times 2$ (internal vs. external, within subjects) repeated measures ANOVA to look for voxels that showed significant differences in activation for object versus attribute and/or internal versus external contrasts. Regions showing significant differences $(p<.05$, uncorrected) are coded by color in Figure 3. Those showing no differences at this threshold are shown in red and are labeled "common," since they respond strongly to switch-no-switch but do not respond preferentially on the basis of type. Responses in the left DLPFC, the IPS, and the striate/ medial extrastriate cortex were most strongly activated by internal switches (blue in Figure 3), whereas the bilateral lateral occipital cortex responded most to external switches (yellow). Medial structures - including the anterior cingulate, the precuneus, and the cerebellar vermis - as well as the left IPS and the premotor cortex, were more strongly activated by object than by attribute shifts (green), and these areas overlapped in the parietal cortex, the left SFS, and the right cerebellum with internal-preferential activations. Few voxels responded more to attribute than to object switches; the strongest effects were in the right sensorimotor cortex and the SFS (cyan). These results demonstrate that there is both substantial commonality among different types of switch costs - notably, even regions that showed preferences for some switch types were nearly always strongly activated by all types, as compared with no-switch trials (Table4) and evidence for switching-type-specific effects. The type-specific effects suggest greater frontal and parietal involvement in switching among WM representations (internal) and greater switching-specific activity in the extrastriate cortex when there is switching among percepts, pointing to a functional role for the extrastriate cortex in switching-specific processing.

Omnibus individual-differences analysis. The results of the omnibus $F$ test for correlations between brain and behavioral switching costs are shown in Figure 4. Panel A shows significant regions with whole-brain FDR correction (yellow; $F>3.52, p<.05$, corrected $/ p<$ .003 , uncorrected) overlaid on horizontal anatomical slices. Regions significantly correlated with behavioral performance in one or more conditions included a num- 
A Regions active in at least two switch types, whole-brain corrected

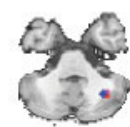

$z=-40$

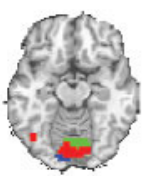

$z=-14$

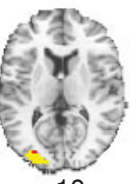

$z=10$

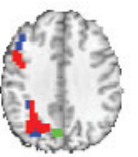

$z=36$

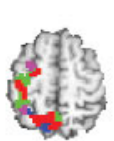

$z=60$

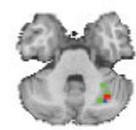

$z=-34$

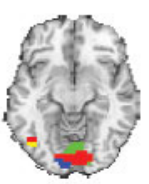

$z=-10$
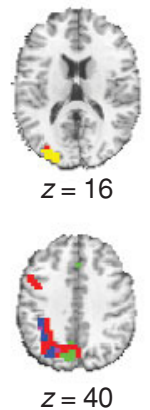
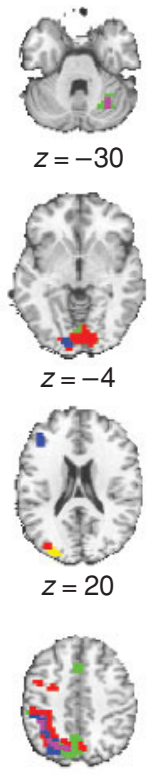

$z=46$

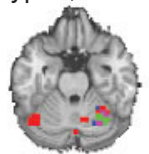

$z=-24$
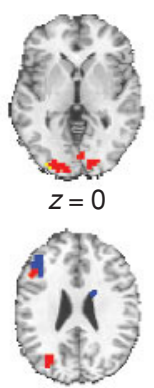

$z=26$

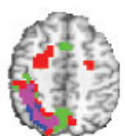

$z=50$
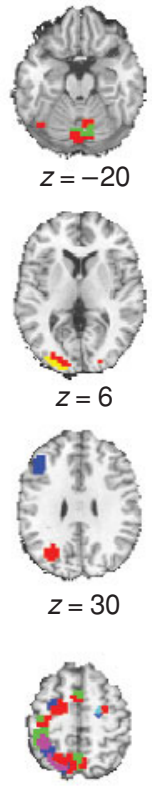

$z=56$

Common activation
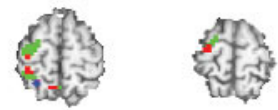

$z=70$

Internal $>$ External

External $>$ Internal

Object $>$ Attribute

Attribute $>$ Object

B Representative slices at $p<.001$, two independent switch types
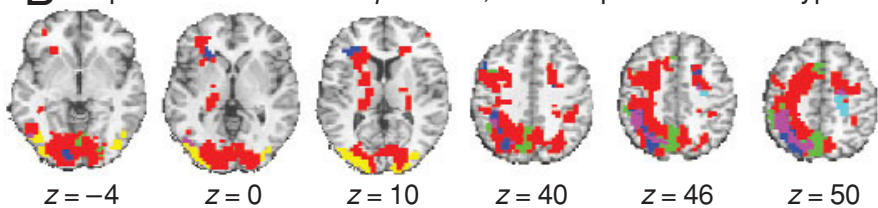

Figure 3. Switch costs in fMRI. All voxels identified show significant switch costs in at least two switch-no-switch contrasts $(p<.05$, corrected in each contrast, in $\mathrm{A}$; and $\boldsymbol{p}<.001$, uncorrected in each contrast, in B). Thus, many regions not shown here may also show brain switch costs at less stringent thresholds. Regions colored in red show no significant differences among costs for different types of switch (at $p<.05$, uncorrected). Other regions show evidence for greater activation in some switch types than in others, as indicated in the legend. Activations were bilateral at lower thresholds. I, internal; E, external; O, object; A, attribute.

ber of cortical regions previously shown to be active in task switching and include essentially the same set of regions as those identified as switching responsive in the previous analysis, with the notable additions of the dorsal and ventral medial PFC. These include the "classical" regions thought to mediate cognitive control: the medial PFC above the cingulate sulcus, the dorsolateral and ventrolateral PFCs, the IPS, and the precuneus on the medial parietal wall. Activation in these areas was leftlateralized, although we found activation bilaterally, and in the anterior cingulate proper, at lower thresholds (e.g., $p<.01$, colored tan in Figure 4A). Figures 4B and 4C (yellow activations) show the significant regions on inflated lateral and medial surfaces, respectively. Figure 4D shows flattened cortical surface representations for the left (left panel) and the right (right panel) hemispheres. Activation coordinates are listed in Table 2. Large clusters of contiguous voxels were separated into subclusters where necessary, and center-of-mass MNI coordinates are reported for each subcluster.

Superimposed on the surface maps in Figure 3D are significant regions from a meta-analysis comparing the density of reported activation peaks in WM tasks that require active manipulation (executive WM) with that for 
Table 4

Switch Costs in Brain Activation

\begin{tabular}{|c|c|c|c|c|c|c|c|c|}
\hline \multirow[b]{2}{*}{ Region } & \multicolumn{3}{|c|}{ MNI Coordinates } & \multirow[b]{2}{*}{ Voxels } & \multicolumn{4}{|c|}{$\underline{\text { Switch Cost Peak } z \text { Scores }}$} \\
\hline & $x$ & $y$ & $z$ & & EO & EA & IO & IA \\
\hline \multicolumn{9}{|l|}{ Frontal cortex } \\
\hline Left premotor & -48 & 2 & 39 & 18 & $2.87^{*}$ & $3.96^{*}$ & $4.67^{*}$ & $3.85^{*}$ \\
\hline prefrontal cortex & -45 & 28 & 27 & 38 & $2.18^{*}$ & $3.83^{*}$ & $5.29^{*}$ & $3.79^{*}$ \\
\hline Left $\mathrm{s}$ & -31 & -12 & 57 & 105 & $4.68^{*}$ & $3.77^{*}$ & $5.05^{*}$ & $4.14^{*}$ \\
\hline Rig & -5 & -10 & 52 & 9 & $2.78^{*}$ & $4.46^{*}$ & $4.45^{*}$ & $4.09^{*}$ \\
\hline Anterior cingula & -2 & 10 & 50 & 19 & $4.29^{*}$ & $2.56^{*}$ & $4.36^{*}$ & $3.14^{*}$ \\
\hline \multicolumn{9}{|l|}{ Parietal cortex } \\
\hline Left precune & -11 & -71 & 50 & 198 & $5.16^{*}$ & $2.99^{*}$ & $4.66^{*}$ & $4.41^{*}$ \\
\hline intraparietal sulcus & -32 & -64 & 43 & 162 & $3.96^{*}$ & $4.50^{*}$ & $5.80^{*}$ & $4.40^{*}$ \\
\hline Left anterior & -41 & -41 & 51 & 121 & $4.09^{*}$ & $4.24^{*}$ & $5.57^{*}$ & $4.08^{*}$ \\
\hline \multicolumn{9}{|l|}{ Basal ganglia } \\
\hline Right caudate & 19 & 0 & 25 & 1 & $1.94^{*}$ & $2.78^{*}$ & $4.26^{*}$ & $3.50^{*}$ \\
\hline \multicolumn{9}{|l|}{ Occipital cortex } \\
\hline Occi & -13 & -92 & -7 & 58 & $4.49^{*}$ & $3.76^{*}$ & $4.32^{*}$ & $4.51^{*}$ \\
\hline Media & 6 & -85 & -11 & 112 & $4.61^{*}$ & $3.07^{*}$ & $4.35^{*}$ & $4.04^{*}$ \\
\hline Left extrastria & -29 & -92 & 11 & 58 & $5.36^{*}$ & $4.84^{*}$ & 1.46 & $3.98^{*}$ \\
\hline Left inferior occipital/cerebellum & -45 & -67 & -19 & 17 & $3.54^{*}$ & $4.53^{*}$ & $2.71^{*}$ & $3.88^{*}$ \\
\hline \multicolumn{9}{|l|}{ Cerebellum } \\
\hline Medial ce & 8 & -65 & -21 & 8 & $2.92^{*}$ & 1.46 & $4.60^{*}$ & $3.71^{*}$ \\
\hline Right superior cerebellum & 30 & -62 & -29 & 30 & $3.98^{*}$ & $2.10^{*}$ & $4.95^{*}$ & $3.79^{*}$ \\
\hline
\end{tabular}

Note-Regions showing whole-brain-corrected switch costs in at least two contrasts. Voxels list the number of voxels meeting this criterion in each region. $x, y$, and $z$ are the Montreal Neurological Institute (MNI) standard brain coordinates for the activation center of mass. EO, external object; EA, external attribute; IO, internal object; IA, internal attribute. ${ }^{*} p<.05$.

A

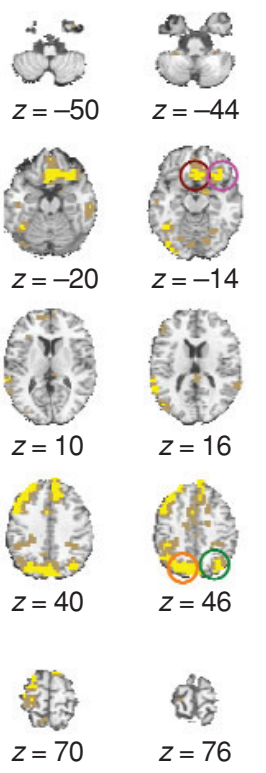

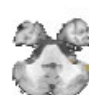

$z=-40$

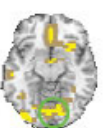

$z=-10$

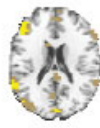

$z=20$

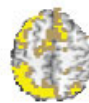

$z=50$

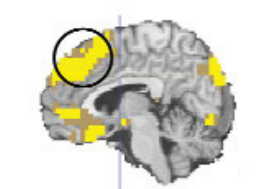

$z=-4$
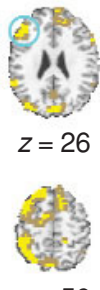

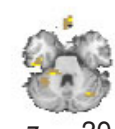

$z=-30$
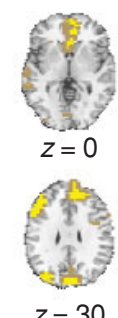

$z=30$

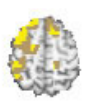

$z=60$
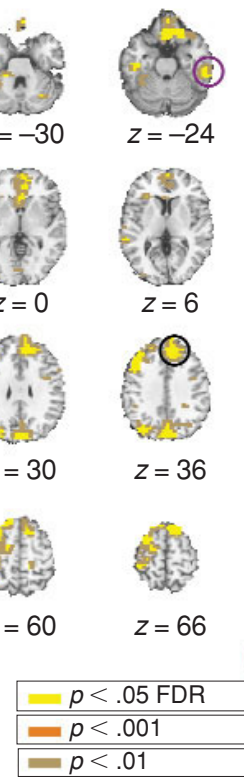

$z=36$

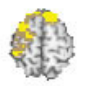

B

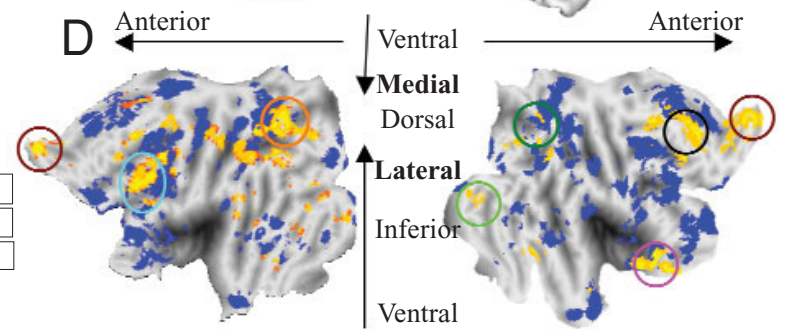

Figure 4. (A) Significant correlations between brain and behavior in the omnibus analysis at $p<.05$, FDR-corrected, shown in yellow, and at $p<.01$, shown in tan. Axial slices are shown in ascending order. (B) Left and right inflated lateral surfaces (left and right panels) with significant activations (corrected) shown in yellow. (C) Medial surfaces. (D) Flattened cortical maps of the left and right hemispheres. Yellow shows significant regions, and blue shows significant meta-analysis results comparing executive working memory with storage-only working memory. Colored circles on the figure identify some landmarks across views in the figure. Circles of the same color surround the same activated region. For flat maps, see http://brainmap.wustl.edu/caret (Van Essen et al., 2001). 


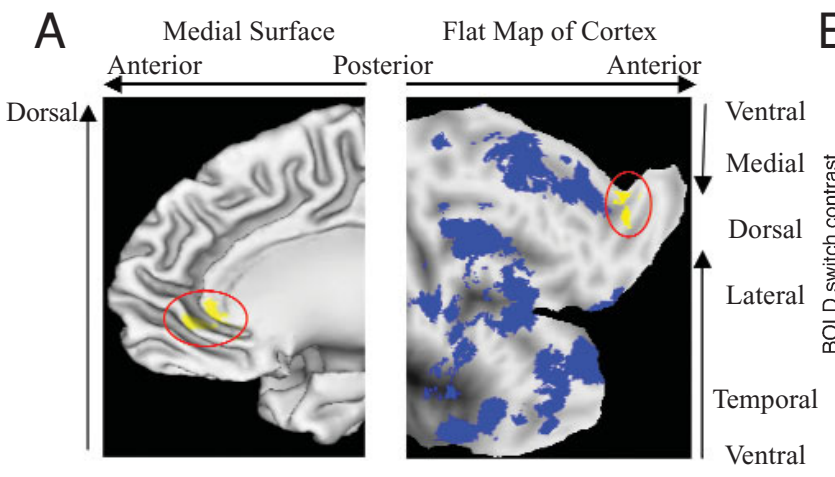

B Brain-behavior correlations

C Correlations With Performance Cost (J2SC)

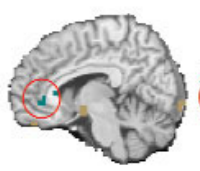

External Object

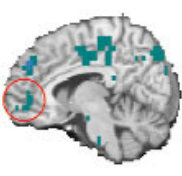

Internal

Object

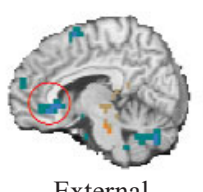

External

Attribute

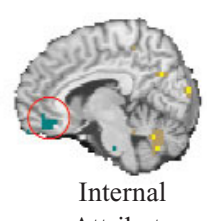

Attribute
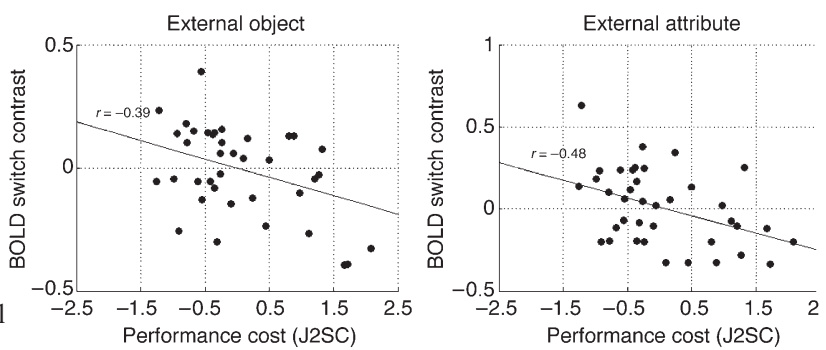

Internal object
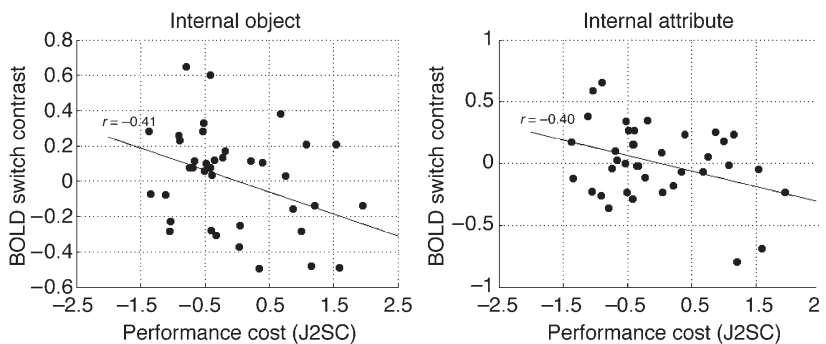

Correlations With Object Switch Time (Cue Period)

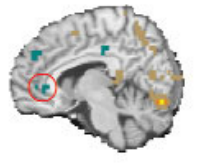

Cue External Object

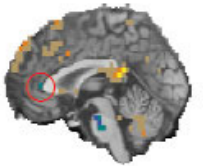

Cue Internal Object

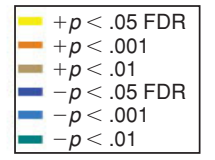

$=-p<.01$

Figure 5. (A) The ventromedial PFC region that showed significant negative correlations between J2 switch costs and each switching contrast with a residual switching component (external object, internal object, external attribute, and internal attribute). A nearly identical region showed negative correlations between cue switch costs and object-switching brain activation (internal object and external object). (B) Scatterplots showing robust partial regression slopes for each brain switch cost. Units are $z$ scores. The shading of each point represents its final weight in the reweighted least-squares analysis; points with dark shading contribute highly, and those with lighter shading are downweighted (black $=$ weight of 1 , white $=$ weight of 0 ). (C) Sagittal slices from individual brain-behavior correlation maps thresholded at $p<.05$, FDR (whole brain); $p<.001 ;$ or $p<.01$. Circles indicate the region in each contrast that was significant in each brain-behavior correlation. As the panels show, some performance-correlated activation in this region was apparent at $p<.01$ in each contrast.

WM tasks that required simple storage in WM (Wager \& Smith, 2003). These regions are shown in blue for comparison with the results of our study. Previous studies have shown two separable medial prefrontal foci for executive WM: one in the mid-cingulate, posterior to the anterior commissure, and one large focus in the dorsal anterior cingulate that may separate into a more rostral and a more caudal focus (Figure 3D). Our results overlap with those for the more caudal focus (smaller activations) bilaterally and the most rostral focus in the right hemisphere.

Notably, behavioral performance also predicted switching activation in ventral areas more often associated with reward, reward-based decision making, and emotion; these areas include the ventromedial PFC (VMPFC), the pregenual anterior cingulate, and the right inferior anterior insula (the agranular insula, likely overlapping with the primary gustatory cortex; Mesulam \& Mufson, 1982). Activation of the agranular insula has been found in many studies of executive working memory (Figure 4D; Wager \& Smith, 2003) and with punishing feedback that leads to a shift in response strategy (O'Doherty, Critchley, Deichmann, \& Dolan, 2003).

Finally, we also found significant effects in regions that have typically been associated with visual processing, including the bilateral extrastriate cortex and the inferior temporal cortex. Superior temporal gyrus activations were found most prominently in the left hemisphere but were bilateral at $p<.001$.

Conjunction analyses. These analyses were directed at determining the pattern of correlations between brain and behavior across conditions, particularly to identify whether regions were positively or negatively correlated with performance and whether they showed patterns of correlations that suggest a role in switching in general, task set or rule engagement components of shifting, or subtypes based on type of representation (object or attribute) or locus of representation (internal/external). 
Rule engagement/general shifting. Most strikingly, the conjunction analysis showed that two regions in the ventromedial PFC and the pregenual anterior cingulate were negatively correlated with shifting performance in each brain contrast that involved rule engagement. This means that the lower the residual shift cost (for objects) or total shift cost (for attributes), the greater the activity in the region. The resulting significant region is shown in Figure 5A, and the individual maps are shown in Figure 5B, thresholded at multiple levels: $p<.05$ (FDR corrected for whole-brain search), $p<.001$, and $p<.01$. The panels in Figure 5B show some results outside the omnibus search mask. Although they may be important for future studies, we restrict our interpretations here to those regions significant in the omnibus analysis. Brainbehavior partial correlation scatterplots for each relevant switch cost are shown in Figure 5C.

The individual switch type analyses showed that better switching performance was related to more ventromedial activation in four of six brain-behavior relationshipsspecifically, those with a rule engagement component. We next performed a post hoc test for whether negative correlations for the other two brain-behavior relationships, which isolated task set shifting among objects, could be found nearby. If so, activation of this area might mediate better overall switching performance, decreasing both performance costs ( $\mathrm{J} 2$ shift cost) and preparatory switch activity (cue shift cost). We searched for voxels within the omnibus region that showed negative correlations between cue shift cost and object switching, significant at $p<.05$ for both IO and EO contrasts. This analysis showed that a region of 7 voxels (equivalently, 62 standard $2 \times 2 \times 2 \mathrm{~mm}$ voxels) in the same region met these criteria, indicating that VMPFC activity is correlated with both task set engagement and rule engagement during switching performance.

No regions showed consistent positive correlations with all behavioral costs involving rule engagement. This was due largely to the object residual shift costs, which showed very little consistent (across internal and external) positive correlation with residual behavioral switch costs. Thus, once preparatory set shifting is accomplished, there is still a residual behavioral RT cost associated with switching. Individuals with high residual RT switch costs show smaller switch-related activations in the VMPFC but do not show larger switch-related activations in the DLPFC, the parietal cortex, and other related regions.

Task set shifting. In a conjunction analysis across shifting brain-behavior correlations that involved preparatory task set engagement (cue shift cost with IO and EO and $\mathrm{J} 2$ shift cost with IA and EA), we found one region that showed significant correlations in all four tests. This region was in the left anterior IPS - a region activated with high reliability in all switch types in the present study and one of the seven regions identified in our previous meta-analysis of task switching (Wager et al., 2004).
A more complete examination of the regions in Table 3 shows that many dorsal cortical regions showed significant positive correlations for preparatory task set switching among objects (cue shift cost with IO and EO) and attribute switching in the internal condition ( $\mathrm{J} 2 \mathrm{shift}$ cost with IA). These regions include the bilateral IPS, the left DLPFC, the precuneus, and the medial PFC, all regions canonically activated in tasks that demand executive processing. One interpretation is that these results reflect switch-type-specific recruitment of an executive attention network, particularly when there is shifting among items in WM (internal) and when objects are selected with multiple associated rules (object). It may be that both these shift types place particularly high demands on manipulation within WM. These findings parallel the greater switch-related activation for internal and object switches in this study, particularly in the left DLPFC, the IPS, and the visual cortex for internal shifting and the precuneus/anterior cingulate for object shifting (Figure2). An additional set of executive regions, including the cerebellum and more of the medial PFC, was positively correlated with switching performance only in internal object (cue shift cost with IO) and attribute (J2 shift cost with IA) shifting. No areas were consistently negatively correlated with task set shifting, apart from the VMPFC region reported above.

\section{DISCUSSION}

We found that switching effects in many of the areas activated by executive processes (Petrides, 1991; Smith \& Jonides, 1999; Wager \& Smith, 2003) were higher for participants who showed high switch RT costs. Most of the regions showing such individual differences were those that were most strongly activated by switching in the group. The anterior IPS, in particular, showed consistently higher activation for rule-engagement-related contrasts in those participants who showed high ruleengagement-related switch costs. The VMPFC, on the other hand, was consistently negatively correlated with switching performance: Better switchers showed more activation in these regions.

How are these brain-behavior correlations to be interpreted? The most straightforward view is that increased dorsal prefrontal and parietal activation reflects the increased time and energy that poor performers spend accomplishing the cognitive operations required to switch attention. This may be because the mechanisms themselves are less efficient (indicating individual differences in a trait ability) or because poor performers employ a strategy that makes switching more difficult. For example, a participant might adopt a strategy (implicit or explicit) of weighting the importance of performance on no-switch trials more heavily, maximizing performance on no-switch trials at the price of increasing switch costs.

The finding of increased switching-related VMPFC activation in better performers is informative for three 
reasons. First, activation in a discrete region of the cortex is related to shifting performance across multiple tasks, providing evidence for some common mechanisms underlying different types of shifting. Second, this result implies that the VMPFC is an important region for task switching, even when switches are guided by explicit task instructions in addition to reward reversals or decisionguided shifts (Fellows \& Farah, 2003; Wallis, Dias, Robbins, \& Roberts, 2001). Third, computational models of task shifting suggest that poor switching is associated with more shift-related activation in regions that represent task sets (e.g., the DLPFC) and with less shiftrelated activation in areas that provide control input to the model. Our finding of less shift-related activation in the VMPFC suggests a conceptual link between this region and control input.

The VMPFC has been associated in the animal literature with updating of the representations of value and reward that are used to guide decisions and/or with inhibition of irrelevant responses after reward contingencies change (Baxter, Parker, Lindner, Izquierdo, \& Murray, 2000; Dias, Robbins, \& Roberts, 1997; Everitt et al., 1999; Wallis et al., 2001). This part of the cortex appears to be necessary when changing reward contingencies signal shifts in attention (Fellows \& Farah, 2003). The idea that changing valuations of stimuli drive shifts in attention provides a natural mechanism for control input in computational models, and our findings are consistent with this view.

However, in our task, reward contingencies are not learned over time but are provided by explicit task instructions. How instructions to attend in a particular way motivate human participants is unclear, but our results suggest that the process of shifting attention on the basis of task instructions is not completely different from shifting attention on the basis of changing reward values. Although the rostral medial PFC has been reported in human studies of instruction-cued attention shifting (Pollmann, 2001; Pollmann, Weidner, Muller, \& von Cramon, 2000; Small etal., 2003), it has not been consistently activated in previous studies in which shift trials have been compared with noshift trials. One potential reason is that if the VMPFC is important for signaling the reward value or behavioral relevance of stimuli, it may not show overall differences in activation between switch and nonswitch trials across all individuals. Rather, this effect might be detectable in an individual-differencess analysis, which examines individual variability in reward-guided relevance assignment during the task.

An important alternative explanation is that this portion of the VMPFC may be part of a default brain network that is active at rest and decreases with cognitive load (see, e.g., Gusnard \& Raichle, 2001). It could be that efficient attention shifters show greater activation in this area because they show smaller cognitive-loaddependent decreases. Although future studies must pit reward-value-related and load-related hypotheses against each other directly, two pieces of evidence from this study argue against the load-related alternative. First, the VMPFC was not consistently deactivated in task switching overall, implying that load-related decreases are not strongly present in this paradigm. Second, the brainbehavior correlations were more reliable in this region than in the dorsal cortex and more consistent across switch types. For the load-dependent decrease hypothesis to be true, the decreases in performance in the VMPFC would have to be more reliable markers of cognitive load than dorsolateral increases are, and the weight of evidence in the literature thus far argues against this point.

\section{Role of the Lateral PFC: Task Representation and Processing Bias}

What do these brain-behavior correlations tell us about the underlying functions of these regions? Presumably, dorsal and ventral regions play dissociable roles in task switching, because of the opposite signs of brainbehavior correlations in the two regions. The dorsolateral PFC is thought to play a central role in the maintenance of goal information (e.g., Asaad, Rainer, \& Miller, 2000). Studies of primate electrophysiology have suggested that neural activity in these areas represents tasks, stimulusresponse mapping rules, and abstract categories of stimuli relevant for behavior. If this is so, why are these regions more active in poorer performers? One account is that these areas work less efficiently in poor performers, requiring greater changes in blood flow to accomplish a maintenance goal. Another explanation is that when control input is deficient, both correct and incorrect task goals are activated, and additional activity is related to this overactivation of multiple tasks while the system activates the appropriate one.

The latter account is elegantly captured in the computational model of Gilbert and Shallice (2002) and in related models (e.g., Cohen, Dunbar, \& McClelland, 1990; Yeung \& Monsell, 2003). In the model, RT switch costs reflect the time it takes to activate network units that represent the correct task units (the model equivalent of the DLPFC). Task units, in turn, strengthen perceptualmotor processing pathways for the correct task and inhibit those related to incorrect tasks. Activation of previous task units persists from trial to trial, so that the incorrect task unit is more activated on switch trials that on nonswitch trials. A control input is provided from outside the model. This control input is presumably related to the decision to perform a particular task. This model explains a number of behavioral findings on RT switching costs, including surprising asymmetries in switch costs thought to be related to persistent activation of incorrect task sets (Allport et al., 1994; Mayr \& Keele, 2000) and the need for control (Yeung \& Monsell, 2003). The model predicts that poorer shifting performance will result in increased activity in task demand regions and response selection regions alike, since incorrect task unit activity is propagated backward to create increased crosstalk throughout the system. 
Table 5

Previous Studies of Emotion (64 Studies), Response Selection (40 Studies), Attention Switching (31 Studies), and Working Memory (60 Studies) That Showed Activation Within 12 mm of the Ventromedial Prefrontal Activations in This Study

\begin{tabular}{|c|c|c|c|c|c|}
\hline \multirow[b]{2}{*}{ Study } & \multicolumn{3}{|c|}{ Coordinates } & \multirow{2}{*}{$\begin{array}{c}\text { Distance } \\
(\mathrm{mm})\end{array}$} & \multirow[b]{2}{*}{ Task } \\
\hline & $x$ & $y$ & $z$ & & \\
\hline \multicolumn{6}{|l|}{ Emotion related } \\
\hline Beauregard et al., 1997 & -8 & 40 & -9 & 9 & passive viewing of emotional vs. neutral words \\
\hline Canli, Desmond, Zhao, Glover, \& Gabrieli, 1998 & 0 & 32 & -15 & 11 & passive viewing of emotional vs. neutral pictures \\
\hline \multirow[t]{4}{*}{ Damasio et al., 2000} & -5 & 35 & 4 & 10 & recall of personal emotional episodes: sad vs. neutral \\
\hline & -1 & 39 & -15 & 11 & recall of personal emotional episodes: sad vs. neutral \\
\hline & 9 & 34 & -10 & 11 & recall of personal emotional episodes: happy vs. neutral \\
\hline & 4 & 38 & 5 & 10 & recall of personal emotional episodes: happy vs. neutral \\
\hline \multirow[t]{3}{*}{ George, Ketter, Parekh, Herscovitch, \& Post, 1996} & -4 & 29 & -5 & 9 & recall of personal events: happy vs. neutral \\
\hline & -6 & 35 & -8 & 7 & recall of personal events: happy vs. neutral \\
\hline & -2 & 37 & 6 & 11 & recall of personal events: happy vs. neutral \\
\hline Liotti et al., 2000 & -2 & 29 & -3 & 9 & recall of personal events: sad vs. neutral \\
\hline Partiot, Grafman, Sadato, Wachs, \& Hallett, 1995 & -2 & 37 & -5 & 2 & imagined responses to a sad vs. neutral hypothetical situation \\
\hline \multirow[t]{2}{*}{ Phillips et al., 1998} & -3 & 43 & 2 & 9 & judging familiarity of happy vs. sad facial expressions \\
\hline & 0 & 38 & 6 & 11 & \\
\hline Kesler-West et al., 2001 & 1 & 47 & -2 & 10 & judging happy vs. neutral faces \\
\hline \multicolumn{6}{|l|}{ Response selection } \\
\hline \multirow[t]{2}{*}{ Peterson et al., 1999} & -8 & 35 & 2 & 11 & Stroop incompatible vs. compatible \\
\hline & 8 & 37 & 2 & 11 & passive viewing of emotional vs. neutral words \\
\hline \multicolumn{6}{|l|}{ Attention shifting } \\
\hline \multicolumn{6}{|l|}{ None (see the text for additional information) } \\
\hline \multicolumn{6}{|l|}{ Working memory } \\
\hline None & & & & & \\
\hline
\end{tabular}

\section{Role of the VMPFC in Computational Models: Relevance and Control Signals}

Interestingly, the model also suggests a role for the VMPFC. If poor performance is associated with increased activity throughout the network, what type of activation is related to good performance? The answer is the control input to task demand units, whose activity increases the efficiency of task selection. The relationship we found between the VMPFC and good performance suggests a role for this region in determining and applying control over which tasks are represented in the DLPFC.

This finding draws the two literatures on the DLPFC and the VMPFC in control and decision making together. How are control inputs configured? Recent modeling efforts have focused on the influence of putatively reward-related dopaminergic projections from the ventral tegmental area to the VMPFC on the task selection process (Braver \& Brown, 2003; Braver \& Cohen, 1997; Cohen, Braver, \& Brown, 2002). The essential idea is that dopamine signals when reward is expected (Schultz, 2000,2002 ) and dopamine input to the VMPFC sends signals that stabilize task representations in WM and facilitate learning. When dopamine input to the VMPFC is reduced - in response to a decrease in the reward value of a choice or stimulus - task representations in the DLPFC are destabilized, and attention shifting occurs.

This process amounts to a rapid updating of the motivational relevance of stimuli or the links between stimuli and expected rewards that determine what one should pay attention to. Animal and human studies support the idea that the VMPFC is critical for updating the current motivational relevance of stimuli (Baxter et al., 2000; O'Doherty, Critchley, et al., 2003; O'Doherty, Dayan, Friston, Critchley, \& Dolan, 2003), and it has been linked by a large literature to mood and motivational disorders (Drevets, 2000, 2001) and affect-guided decision making (Bechara, Damasio, Damasio, \& Anderson, 1994; Bechara, Damasio, Tranel, \& Damasio, 1997). Table 5 shows some other studies that have reported activation foci within $12 \mathrm{~mm}$ of the centroid in our VMPFC results. The studies were drawn from a pool of 64 emotion studies, 31 shifting studies, 60 working memory studies, and 37 response interference studies. The table shows that a number of emotion-related studies in healthy humans produced VMPFC activation overlapping with our results and that most of these involved recall of personally salient emotional events. Strikingly, no studies of attention switching (out of the 31 we reviewed) or executive WM did (but see Pollmann, 2001; Small etal., 2003). Thus, our highly reliable individual-differences effects in task switching in this region provide new information about the role the VMPFC plays in attention switching, and the broad role of the VMPFC in affective and motivational processes suggest that its role in switching is also similar (Baxter et al., 2000; O'Doherty, Critchley, et al., 2003).

Taken as a whole, the evidence is consistent with the interpretation that the VMPFC plays a role in task switching: specifically, it may be involved in the updating of motivational relevance of stimuli when a participant is instructed to switch attention. These relevance signals, in turn, activate task demand representations in the PFC that bias perceptual processing and enact a shift. 
These interpretations lead us to one additional speculative conclusion: Efficient updating of motivational value may be a key difference between those who show high and those who show low shift costs.

\section{REFERENCES}

Allport, A., Styles, E. A., \& Hsieh, S.L. (1994). Shifting intentional set: Exploring the dynamic control of tasks. In C. Umiltà \& M. Moscovitch (Eds.), Attention and performance XV: Conscious and nonconscious information processing (pp. 421-452). Cambridge, MA: MIT Press, Bradford Books.

Anderson, J. R., \& LeBiere, C. (1998). The atomic components of thought. Mahwah, NJ: Erlbaum.

Asaad, W. F., Rainer, G., \& Miller, E. K. (2000). Task-specific neural activity in the primate prefrontal cortex. Journal of Neurophysiology, 84, 451-459.

Ashburner, J., \& Friston, K. J. (1997). Spatial transformation of images. In R. S. J. Frackowiak, K. J. Friston, C. D. Frith, R. J. Dolan, \& J.C. Mazziotta (Eds.), Human brain function (pp. 43-59). San Diego: Academic Press.

BADDELEY, A. (1992). Working memory. Science, 255, 556-559.

Baxter, M. G., Parker, A., Lindner, C. C., Izquierdo, A. D., \& Murray, E. A. (2000). Control of response selection by reinforcer value requires interaction of amygdala and orbital prefrontal cortex. Journal of Neuroscience, 20, 4311-4319.

Beauregard, M., Chertkow, H., Bub, D., Murtha, S., Dixon, R., \& Evans, A. (1997). The neural substrate for concrete, abstract, and emotional word lexica: A positron emission tomography study. Journal of Cognitive Neuroscience, 9, 441-461.

Bechara, A., Damasio, A. R., Damasio, H., \& Anderson, S. W. (1994). Insensitivity to future consequences following damage to human prefrontal cortex. Cognition, 50, 7-15.

Bechara, A., Damasio, H., Tranel, D., \& Damasio, A. R. (1997). Deciding advantageously before knowing the advantageous strategy. Science, 275, 1293-1295.

Bleckley, M. K., Durso, F. T., Crutchfield, J. M., Engle, R. W., \& Khanna, M. M. (2003). Individual differences in working memory capacity predict visual attention allocation. Psychonomic Bulletin \& Review, 10, 884-889.

Braver, T. S., \& Brown, J. W. (2003). Principles of pleasure prediction: Specifying the neural dynamics of human reward learning. $\mathrm{Neu}$ ron, 38, 150-152.

Braver, T. S., \& Cohen, J. D. (1997). An integrated computational model of dopamine function in reinforcement learning and working memory. Society for Neuroscience Abstracts, 23, 775.

Canli, T., Desmond, J. E., Zhao, Z., Glover, G., \& Gabrieli, J. D. (1998). Hemispheric asymmetry for emotional stimuli detected with fMRI. NeuroReport, 9, 3233-3239.

Cohen, J. D., Braver, T. S., \& Brown, J. W. (2002). Computational perspectives on dopamine function in prefrontal cortex. Current Opinion in Neurobiology, 12, 223-229.

Cohen, J. D., Dunbar, K., \& McClelland, J. L. (1990). On the control of automatic processes: A parallel distributed processing account of the Stroop effect. Psychological Review, 97, 332-361.

Conway, A. R. A., Kane, M. J., \& Engle, R. W. (2003). Working memory capacity and its relation to general intelligence. Trends in Cognitive Sciences, 7, 547-552.

Coull, J. T., Frith, C. D., Buchel, C., \& Nobre, A. C. (2000). Orienting attention in time: Behavioural and neuroanatomical distinction between exogenous and endogenous shifts. Neuropsychologia, 38, 808-819.

Damasio, A. R., Grabowski, T. J., Bechara, A., Damasio, H., Ponto, L. L., Parvizi, J., \& Hichwa, R. D. (2000). Subcortical and cortical brain activity during the feeling of self-generated emotions. Nature Neuroscience, 3, 1049-1056.

Della-Maggiore, V., Chau, W., Peres-Neto, P. R., \& Mcintosh, A. R. (2002). An empirical comparison of SPM preprocessing parameters to the analysis of fMRI data. NeuroImage, 17, 19-28.

Dias, R., Robbins, T. W., \& Roberts, A. C. (1997). Dissociable forms of inhibitory control within prefrontal cortex with an analog of the Wisconsin Card Sort Test: Restriction to novel situations and independence from "on-line" processing. Journal of Neuroscience, 17, 9285-9297.

Drevets, W. C. (2000). Neuroimaging studies of mood disorders. Biological Psychiatry, 48, 813-829.

Drevets, W. C. (2001). Neuroimaging and neuropathological studies of depression: Implications for the cognitive-emotional features of mood disorders. Current Opinion in Neurobiology, 11, 240-249.

Everitt, B. J., Parkinson, J. A., Olmstead, M. C., Arroyo, M., RobLEDO, P., \& RoBbins, T. W. (1999). Associative processes in addiction and reward: The role of amygdala-ventral striatal subsystems. In J. F. McGinty (Ed.), Advancing from the ventral striatum to the extended amygdala: Implications for neuropsychiatry and drug abuse (Annals of the New York Academy of Sciences, Vol. 877, pp. 412438). New York: New York Academy of Sciences.

Fellows, L. K., \& Farah, M. J. (2003). Ventromedial frontal cortex mediates affective shifting in humans: Evidence from a reversal learning paradigm. Brain, 126, 1830-1837.

Garavan, H. (1998). Serial attention within working memory. Memory \& Cognition, 26, 263-276.

Gavrilescu, M., Shaw, M. E., Stuart, G. W., Eckersley, P., Svalbe, I. D., \& EGAN, G. F. (2002). Simulation of the effects of global normalization procedures in functional MRI. NeuroImage, 17, 532542.

Genovese, C. R., Lazar, N. A., \& Nichols, T. (2002). Thresholding of statistical maps in functional neuroimaging using the false discovery rate. NeuroImage, $\mathbf{1 5}, 870-878$.

George, M. S., Ketter, T. A., Parekh, P. I., Herscovitch, P., \& Post, R. M. (1996). Gender differences in regional cerebral blood flow during transient self-induced sadness or happiness. Biological Psychiatry, 40, 859-871.

Gilbert, S. J., \& Shallice, T. (2002). Task switching: A PDP model. Cognitive Psychology, 44, 297-337.

Gopher, D., Armony, L., \& GreenshPan, Y. (2000). Switching tasks and attention policies. Journal of Experimental Psychology: General, 129, 308-339.

Gusnard, D. A., \& Raichle, M.E. (2001). Searching for a baseline: Functional imaging and the resting human brain. Nature Reviews Neuroscience, 2, 685-694.

HsieH, S., \& Allport, A. (1994). Shifting attention in a rapid visualsearch paradigm. Perceptual \& Motor Skills, 79, 315-335.

Jenkinson, M., Bannister, P., Brady, M., \& Smith, S. (2002). Improved optimization for the robust and accurate linear registration and motion correction of brain images. NeuroImage, 17, 825-841.

KANE, M. J., \& ENGLE, R. W. (2003). Working-memory capacity and the control of attention: The contributions of goal neglect, response competition, and task set to Stroop interference. Journal of Experimental Psychology: General, 132, 47-70.

Kane, M. J., Hambrick, D.Z., Tuholski, S. W., Wilhelm, O., Payne, T. W., \& ENGLE, R. W. (2004). The generality of working memory capacity: A latent-variable approach to verbal and visuospatial memory span and reasoning. Journal of Experimental Psychology: General, 133, 189-217.

Kesler-West, M. L., Andersen, A. H., Smith, C.D., Avison, M. J., Davis, C.E., Kryscio, R. J., \& Blonder, L.X. (2001). Neural substrates of facial emotion processing using fMRI. Cognitive Brain Research, 11, 213-226.

Liotti, M., Mayberg, H. S., Brannan, S. K., McGinnis, S., JerABEK, P., \& Fox, P. T. (2000). Differential limbic-cortical correlates of sadness and anxiety in healthy subjects: Implications for affective disorders. Biological Psychiatry, 48, 30-42.

Loftus, G. R., \& MAsson, M.E.J. (1994). Using confidence-intervals in within-subject designs. Psychonomic Bulletin \& Review, 1, 476-490.

MAYR, U. (2003). Towards principles of executive control: How mental sets are selected. In R. H. Kluwe, G. Luer, \& F. Rosler (Eds.), Principles of learning and memory (Vol. 1, pp. 223-241). Basel: Birkhauser.

MaYr, U., \& Keele, S.W. (2000). Changing internal constraints on action: The role of backward inhibition. Journal of Experimental Psychology: General, 129, 4-26. 
Mayr, U., \& Kliegl, R. (2000). Task-set switching and long-term memory retrieval. Journal of Experimental Psychology: Learning, Memory, \& Cognition, 26, 1124-1140.

Meiran, N. (1996). Reconfiguration of processing mode prior to task performance. Journal of Experimental Psychology: Learning, Memory, \& Cognition, 22, 1423-1442.

Meiran, N., Chorev, Z., \& SAPIR, A. (2000). Component processes in task switching. Cognitive Psychology, 41, 211-253.

Meiran, N., Hommel, B., Bibi, U., \& Lev, I. (2002). Consciousness and control in task switching. Consciousness \& Cognition, 11, 10-33.

Mesulam, M. M., \& Mufson, E. J. (1982). Insula of the old world monkey: I. Architectonics in the insulo-orbito-temporal component of the paralimbic brain. Journal of Comparative Neurology, 212, 1-22.

Meyer, D. E., \&. Kieras, D.E. (1997). A computational theory of executive cognitive processes and multiple-task perfomance: Pt. 1. Basic mechanisms. Psychological Review, 104, 3-65.

Miyake, A., Friedman, N. P., Emerson, M. J., Witzki, A. H., HowERTER, A., \& WAGER, T.D. (2000). The unity and diversity of executive functions and their contributions to complex "frontal lobe" tasks: A latent variable analysis. Cognitive Psychology, 41, 49-100.

Monsell, S., Yeung, N., \& Azuma, R. (2000). Reconfiguration of task-set: Is it easier to switch to the weaker task? Psychological Research/Psychologische Forschung, 63, 250-264.

Newell, A. (1992). Precis of unified theories of cognition. Behavioral \& Brain Sciences, 15, 425-437.

O’Doherty, J. [P.], Critchley, H., Deichmann, R., \& Dolan, R. J. (2003). Dissociating valence of outcome from behavioral control in human orbital and ventral prefrontal cortices. Journal of Neuroscience, 23, 7931-7939.

O’Doherty, J. P., Dayan, P., Friston, K., Critchley, H., \& DoLAN, R. J. (2003). Temporal difference models and reward-related learning in the human brain. Neuron, 38, 329-337.

OpPenheIM, A. V., Schafer, R. W., \& BucK, J.R. (1999). Discrete-time signal processing (2nd ed.). Upper Saddle River, NJ: Prentice-Hall.

Owen, A. M., Roberts, A. C., Polkey, C.E., Sahakian, B. J., \& RobBINS, T. W. (1991). Extra-dimensional versus intra-dimensional set shifting performance following frontal lobe excisions, temporal lobe excisions or amygdalo-hippocampectomy in man. Neuropsychologia, 29, 993-1006

Partiot, A., Grafman, J., Sadato, N., Wachs, J., \& Hallett, M. (1995). Brain activation during the generation of non-emotional and emotional plans. NeuroReport, 6, 1397-1400.

Peterson, B. S., Skudlarski, P., Gatenby, J. C., Zhang, H., AnderSON, A. W., \& GoRE, J. C. (1999). An fMRI study of Stroop wordcolor interference: Evidence for cingulate subregions subserving multiple distributed attentional systems. Biological Psychiatry, 45, 1237-1258.

Petrides, M. (1991). Functional specialization within the dorsolateral frontal cortex for serial order memory. Proceedings of the Royal Society of London: Series B, 246, 299-306.

Phillips, M. L., Bullmore, E. T., Howard, R., Woodruff, P. W., Wright, I. C., Williams, S. C., Simmons, A., Andrew, C., BramMER, M., \& DAVID, A. S. (1998). Investigation of facial recognition memory and happy and sad facial expression perception: An fMRI study. Psychiatry Research, 83, 127-138.

Pollmann, S. (2001). Switching between dimensions, locations, and responses: The role of the left frontopolar cortex. NeuroImage, 14, S118-S124.

Pollmann, S., Weidner, R., Müller, H. J., \& von Cramon, D. Y. (2000). A fronto-posterior network involved in visual dimension changes. Journal of Cognitive Neuroscience, 12, 480-494.

Posner, M. I., \& Rothbart, M. K. (1998). Attention, self-regulation and consciousness. Philosophical Transactions of the Royal Society of London: Series B, 353, 1915-1927.

Rogers, R.D., \& Monsell, S. (1995). Costs of a predictable switch between simple cognitive tasks. Journal of Experimental Psychology: General, 124, 207-231.

Rubinstein, J.S., Meyer, D.E., \& Evans, J.E. (2001). Executive control of cognitive processes in task switching. Journal of Experimental Psychology: Human Perception \& Performance, 27, 763-797.
Salthouse, T.A. (1996). The processing-speed theory of adult age differences in cognition. Psychological Review, 103, 403-428.

Salthouse, T. A., Fristoe, N., McGuthry, K. E., \& Hambrick, D.Z. (1998). Relation of task switching to speed, age, and fluid intelligence. Psychology \& Aging, 13, 445-461.

Schultz, W. (2000). Multiple reward signals in the brain. Nature Reviews Neuroscience, 1, 199-207.

Schultz, W. (2002). Getting formal with dopamine and reward. Neuron, 36, 241-263.

Shafiullah, M., \& Monsell, S. (1999). The cost of switching between Kanji and Kana while reading Japanese. Language \& Cognitive Processes, 14, 567-607.

Shallice, T., \& Burgess, P. (1996). The domain of supervisory processes and temporal organization of behaviour. Philosophical Transactions of the Royal Society of London: Series B, 351, 1405-1411; discussion, 1411-1412

Small, D. M., Gitelman, D. R., Gregory, M.D., Nobre, A. C., ParRISH, T. B., \& Mesulam, M. M. (2003). The posterior cingulate and medial prefrontal cortex mediate the anticipatory allocation of spatial attention. NeuroImage, 18, 633-641.

Smith, E. E., \& Jonides, J. (1999). Storage and executive processes in the frontal lobes. Science, 283, 1657-1661.

Spector, A., \& Biederman, I. (1976). Mental set and mental shift revisited. American Journal of Psychology, 89, 669-679.

Sylvester, C. Y. C., Wager, T. D., LaCey, S. C., Hernandez, L., Nichols, T.E., SMith, E.E., \& Jonides, J. (2003). Switching attention and resolving interference: fMRI measures of executive functions. Neuropsychologia, 41, 357-370.

Van Essen, D. C., Dickson, J., Harwell, J., Hanlon, D., Anderson, C. H., \& DruRY, H. A. (2001). An integrated software system for surface-based analyses of cerebral cortex. Journal of the American Medical Informatics Association, 41, 1359-1378.

Wager, T. D., Keller, M. C., Lacey, S. C., \& Jonides, J. (2005). Increased sensitivity in neuroimaging analyses using robust regression. NeuroImage, 26, 99-113.

WAGER, T.D., \& Nichols, T.E. (2003). Optimization of experimental design in fMRI: A general framework using a genetic algorithm. NeuroImage, 18, 293-309.

WAGER, T.D., READING, S., \& Jonides, J. (2004). Neuroimaging studies of shifting attention: A meta-analysis. NeuroImage, 22, 1679-1693.

WAGER, T.D., \& SMiTH, E.E. (2003). Neuroimaging studies of working memory: A meta-analysis. Cognitive, Affective, \& Behavioral Neuroscience, 3, 255-274.

Wallis, J.D., Dias, R., Robbins, T. W., \& RoberTs, A.C. (2001). Dissociable contributions of the orbitofrontal and lateral prefrontal cortex of the marmoset to performance on a detour reaching task. European Journal of Neuroscience, 13, 1797-1808.

Yeung, N., \& Monsell, S. (2003). Switching between tasks of unequal familiarity: The role of stimulus-attribute and response-set selection. Journal of Experimental Psychology: Human Perception \& Performance, 29, 455-469.

\section{NOTE}

1. One question is whether both readiness responses and a long CTI allow a participant to prepare equivalently for a new task (i.e., whether cue switch costs reflect endogenous switching). Meiran et al. (2002) found that long readiness times decrease switch costs by a small amount but that the benefit is much less than that from a very long CTI. This suggests that participants use the cue period to prepare but respond before they are fully prepared. However, our cue switch costs are an order of magnitude longer than Meiran etal.'s readiness response times, which may reflect greater use of the cue due to enhanced motivation to prepare in our participants: We paid participants block by block on the basis of a combination of overall accuracy and continuing improvements in speed.

(Manuscript received October 4, 2004; revision accepted for publication March 28, 2005.) 